\title{
Polarization chaos and random bit generation in nonlinear fiber optics induced by a time- delayed counter-propagating feedback loop
}

\author{
J. Morosi, ${ }^{1,2}$ N. Berti, ${ }^{1}$ A. Akrout, ${ }^{1}$ A. PicozzI, ${ }^{1}$ M. Guasoni, ${ }^{3}$ AND J. \\ FATOME ${ }^{1, *}$ \\ ${ }^{1}$ Laboratoire Interdisciplinaire Carnot de Bourgogne (ICB), UMR 6303 CNRS-Université de Bourgogne \\ Franche-Comté, 9 av. A. Savary, 21078 Dijon, France \\ ${ }^{2}$ Politecnico di Milano, DEIB-POLICOM Lab, Via Ponzio 34/5, 20133 Milan, Italy \\ ${ }^{3}$ Optoelectronics Research Centre, University of Southampton, SO17 1BJ, UK \\ jfatome@u-bourgogne.fr
}

\begin{abstract}
In this manuscript, we experimentally and numerically investigate the chaotic dynamics of the state-of-polarization in a nonlinear optical fiber due to the cross-interaction between an incident signal and its intense backward replica generated at the fiber-end through an amplified reflective delayed loop. Thanks to the cross-polarization interaction between the two-delayed counter-propagating waves, the output polarization exhibits fast temporal chaotic dynamics, which enable a powerful scrambling process with moving speeds up to $600-\mathrm{krad} / \mathrm{s}$. The performance of this all-optical scrambler was then evaluated on a $10-\mathrm{Gbit} / \mathrm{s}$ On/Off Keying telecom signal achieving an error-free transmission. We also describe how these temporal and chaotic polarization fluctuations can be exploited as an all-optical random number generator. To this aim, a billion-bit sequence was experimentally generated and successfully confronted to the dieharder benchmarking statistic tools. Our experimental analysis are supported by numerical simulations based on the resolution of counterpropagating coupled nonlinear propagation equations that confirm the observed behaviors.
\end{abstract}

(C) 2018 Optical Society of America under the terms of the OSA Open Access Publishing Agreement

OCIS codes: (060.4370) Nonlinear optics, fibers; (070.4340) Nonlinear optical signal processing; (230.4320) Nonlinear optical devices.

\section{References and links}

1. A. Argyris, D. Syvridis, L. Larger, V. Annovazzi-Lodi, P. Colet, I. Fischer, J. García-Ojalvo, C. R. Mirasso, L. Pesquera, and K. A. Shore, "Chaos-based communications at high bit rates using commercial fibre-optic links," Nature 438(7066), 343-346 (2005).

2. L. Keuninckx, M. C. Soriano, I. Fischer, C. R. Mirasso, R. M. Nguimdo, and G. Van der Sande, "Encryption key distribution via chaos synchronization," Sci. Rep. 7, 43428 (2017).

3. L. Larger and J. M. Dudley, "Nonlinear dynamics: Optoelectronic chaos," Nature 465(7294), 41-42 (2010).

4. D. Sussillo and L. F. Abbott, "Generating coherent patterns of activity from chaotic neural networks," Neuron 63(4), 544-557 (2009).

5. F. Duport, A. Smerieri, A. Akrout, M. Haelterman, and S. Massar, "Fully analogue photonic reservoir computer," Sci. Rep. 6(1), 22381 (2016).

6. A. Uchida, K. Amano, M. Inoue, K. Hirano, S. Naito, H. Someya, I. Oowada, T. Kurashige, M. Shiki, S. Yoshimori, K. Yoshimura, and P. Davis, "Fast physical random bit generation with chaotic semiconductor lasers," Nat. Photonics 2(12), 728-732 (2008).

7. I. Kanter, Y. Aviad, I. Reidler, E. Cohen, and M. Rosenbluh, “An optical ultrafast random bit generator," Nat. Photonics 4(1), 58-61 (2010).

8. M. Virte, K. Panajotov, H. Thienpont, and M. Sciamanna, "Deterministic polarization chaos from a laser diode," Nat. Photonics 7(1), 60-65 (2013).

9. B. Wetzel, K. J. Blow, S. K. Turitsyn, G. Millot, L. Larger, and J. M. Dudley, "Random walks and random numbers from supercontinuum generation," Opt. Express 20(10), 11143-11152 (2012).

10. C. Gabriel, C. Wittmann, D. Sych, R. Dong, W. Mauerer, U. L. Andersen, C. Marquardt, and G. Leuchs, "A generator for unique quantum random numbers based on vacuum states," Nat. Photonics 4(10), 711-715 (2010).

11. C. Wu, B. Bai, Y. Liu, X. Zhang, M. Yang, Y. Cao, J. Wang, S. Zhang, H. Zhou, X. Shi, X. Ma, J. G. Ren, J. Zhang, C. Z. Peng, J. Fan, Q. Zhang, and J. W. Pan, "Random Number Generation with Cosmic Photons," Phys. Rev. Lett. 118(14), 140402 (2017). 
12. C. R. S. Williams, J. C. Salevan, X. Li, R. Roy, and T. E. Murphy, "Fast physical random number generator using amplified spontaneous emission,” Opt. Express 18(23), 23584-23597 (2010).

13. X. Li, A. B. Cohen, T. E. Murphy, and R. Roy, "Scalable parallel physical random number generator based on a superluminescent LED," Opt. Lett. 36(6), 1020-1022 (2011).

14. B. Sanguinetti, A. Martin, H. Zbinden, and N. Gisin, "Quantum random number generation on a mobile phone," Phys. Rev. X 4(3), 031056 (2014).

15. T. Lunghi, J. B. Brask, C. C. W. Lim, Q. Lavigne, J. Bowles, A. Martin, H. Zbinden, and N. Brunner, "Selftesting quantum random number generator," Phys. Rev. Lett. 114(15), 150501 (2015).

16. F. Bruyere, O. Audouin, V. Letellier, G. Bassier, and P. Marmier, "Demonstration of an optimal polarization scrambler for long-haul optical amplifier systems," IEEE Photonics Technol. Lett. 6(9), 1153-1155 (1994).

17. Z. Li, J. Mo, Y. Wang, and C. Lu, "Experimental evaluation of the e ect of polarization scrambling speed on the performance of PMD mitigation using FEC," in Optical Fiber Commun. Conference (2004).

18. R. Noé, B. Koch, D. Sandel, and V. Mirvoda, "100-krad/s endless polarisation tracking with miniaturised module card," Electron. Lett. 47(14), 813-814 (2011).

19. W. H. J. Aarts and G. Khoe, "New endless polarization control method using three fiber squeezers," J. Lightwave Technol. 7(7), 1033-1043 (1989).

20. P. Boffi, M. Ferrario, L. Marazzi, P. Martelli, P. Parolari, A. Righetti, R. Siano, and M. Martinelli, "Stable 100$\mathrm{Gb} / \mathrm{s}$ POLMUX-DQPSK transmission with automatic polarization stabilization," IEEE Photonics Technol. Lett. 21(11), 745-747 (2009).

21. Y. K. Lizé, R. Gomma, R. Kashyap, L. Palmer, and A. E. Willner, "Fast all-fiber polarization scrambling using re-entrant Lefèvre controller," Opt. Commun. 279(1), 50-52 (2007).

22. F. Heismann, "Compact electro-optic polarization scramblers for optically amplified lightwave systems," J. Lightwave Technol. 14(8), 1801-1814 (1996).

23. J.-W. Kim, S.-H. C. W.-S. Park, W. S. Chu, and M. C. Oh, "Integrated-optic polarization controllers incorporating polymer waveguide birefringence modulators," Opt. Express 20(11), 12443-12448 (2012).

24. M. Guasoni, P. Y. Bony, M. Gilles, A. Picozzi, and J. Fatome, "Fast and Chaotic Fiber-Based Nonlinear Polarization Scrambler," IEEE J. Sel. Top. Quantum Electron. 22(2), 88 (2016).

25. P.-Y. Bony, M. Guasoni, P. Morin, D. Sugny, A. Picozzi, H. R. Jauslin, S. Pitois, and J. Fatome, "Temporal spying and concealing process in fibre-optic data transmission systems through polarization bypass," Nat. Commun. 5, 4678 (2014).

26. J. Fatome, S. Pitois, P. Morin, E. Assémat, D. Sugny, A. Picozzi, H. R. Jauslin, G. Millot, V. V. Kozlov, and S. Wabnitz, “A universal optical all-fiber Omnipolarizer," Sci. Rep. 2(1), 938 (2012).

27. P.-Y. Bony, M. Guasoni, E. Assémat, S. Pitois, D. Sugny, A. Picozzi, H. R. Jauslin, and J. Fatome, "Optical flipflop memory and data packet switching operation based on polarization bistability in a telecomunication optical fiber,” J. Opt. Soc. Am. B 30(8), 2318-2325 (2013).

28. J. Morosi, A. Akrout, A. Picozzi, M. Gilles, M. Guasoni, and J. Fatome, "Polarization Chaos in Nonlinear Optical Fibers Induced by a Reflective Delayed Loop," in Conference on Lasers and Electro-Optics, OSA Technical Digest (online) (Optical Society of America, 2017), paper SM2M.3.

29. http://www.phy.duke.edu/ rgb/General/dieharder.php

30. V. Kozlov, J. Nuño, and S. Wabnitz, "Theory of lossless polarization attraction in telecommunication fibers," J. Opt. Soc. Am. B 28(1), 100-108 (2011).

31. M. Guasoni, J. Garnier, B. Rumpf, D. Sugny, J. Fatome, F. Amrani, G. Millot, and A. Picozzi, "Incoherent Fermi-Pasta-Ulam recurrences and unconstrained thermalization mediated by strong phase-correlations," Phys. Rev. X 7(1), 011025 (2017).

32. M. T. Rosenstein, J. J. Collins, and C. J. A. De Luca, "Practical method for calculating largest Lyapunov exponents from small data sets," Physica D 65(1-2), 117-134 (1993).

33. Dataset 1: Results of the dieharder tests for the experimental 1-billion bit sequence generated from polarization chaos https://doi.org/10.6084/m9.figshare.5725807.

\section{Introduction}

Nowadays, chaotic dynamics in optical systems find numerous applications, among which the most emblematic are cryptographic secure communications [1-3], reservoir computing [4,5] or random bit generation [6-15]. Similarly to other physical areas, the common feature of those systems is that the route to chaos is achieved by means of a time-delayed feedback. In this contribution, we particularly focus our attention on the chaotic dynamics of the polarization state occurring in optical fiber and driven by a counter-propagating feedback setup. Beyond its fundamental aspect, understanding and harvesting the randomness of the light state-of-polarization (SOP) can find several practical applications. For instance, polarization scrambler devices are mainly implemented in optical communication and exploit polarization randomness in order to ensure polarization diversity in transmission links so as to mitigate the impact of polarization mode dispersion [16-17]. There are also mandatory apparatus when testing the performances of polarization-sensitive fiber devices, integrated 
systems or optical components. While commercially available polarization scramblers are usually based on the cascade of fiber resonant coils, rotating wave-plates, or fiber squeezers and opto-electronic elements [18-23], we have recently introduced an alternative approach based on the nonlinear Kerr effect occurring in optical fibers [24,25]. Fundamentally, it relies on an additional operating mode, i.e. the chaotic regime, of a bistable device called Omnipolarizer, originally designed to operate as an all-optical polarization funnel [26,27]. The principle of operation consists in an incident signal with a fixed polarization-state which nonlinearly interacts through a cross-polarization process with its own backward replica generated at the fiber-end thanks to an amplified reflective loop. When a strong power imbalance between the two counter-propagating waves is applied, the system becomes unstable and chaotic temporal fluctuations of the output SOP are then observed, thus leading to an optical scrambling system [24]. In this new contribution, we go significantly beyond our previous works and show that the polarization instability and its route to chaos can be greatly improved by feeding this self-organized system with an optical delay [28]. In particular, the instability threshold and transient regimes leading to a chaotic regime have been found to be greatly reduced when a time-delayed feedback is incorporated in the system with a time scale larger than the characteristic nonlinear time. We then report on a series of experiments involving a 1-km long highly nonlinear fiber (HNLF) and show that scrambling speeds up to $600-\mathrm{krad} / \mathrm{s}$ can be achieved. Moreover, a 2.5 -reduction in terms of power threshold compared to our previous results reported in [24] as well as a dramatic reduction of fluctuations in the scrambling speed and output degree-of-polarization (DOP) have been observed. Experimental measurements are well confirmed by numerical predictions based on the numerical resolution of a set of counter-propagating nonlinear propagation equations. The performance of this alloptical chaotic scrambler has then been evaluated for telecom applications on a 10-Gbit/s On/Off Keying (OOK) signal showing that an error-free transmission can be achieved in a fully chaotic scrambling regime.

The second practical application highlighted in this contribution relies on the possibility for a chaotic system to harvest its randomness properties in order to generate random numbers [6-13]. Indeed, a genuine random number generator (RNG) must produce unpredictable, unreproducible and unbiased sequences of numbers. For that specific reason, many true RNGs are based on the peculiar properties that characterize chaotic dynamics. Practically, the advantage of using an optical approach is that one can generate random numbers at high repetition rate directly in the physical layer rather than using classical algorithmic techniques. Examples of such all-optical RNG include optoelectronic devices such as chaotic oscillations of high-bandwidth lasers [6,7], polarization chaos from a VCSEL diode [8], supercontinuum generation [9], homodyne detection of vacuum states [10], cosmic photons [11], spontaneous emission [12], superluminescent diodes [13] or exploiting the randomness inherent to quantum mechanics effects $[14,15]$. In the last section of this manuscript, we propose to exploit the chaotic SOP dynamics induced by the time-delayed feedback loop to generate random binary sequences. In this proof-of-concept experiment, the evolution over time of the output Stokes parameters has been recorded and sampled according to a fixed threshold so as to compute a binary sequence of one billion of bits. The degree of randomness of the generated bit sequence has been evaluated using the standard statistical benchmark provided by the dieharder testing suite [29], which shows that polarization chaos in optical fiber can be an efficient source of randomness for the generation of random numbers.

\section{Principle and modeling}

The system under-study is depicted in Fig. 1. It basically consists in a nonlinear Kerr medium, here a segment of randomly birefringent telecommunication fiber of length $L$, in which an initial forward signal defined by its Stokes vector $\boldsymbol{S}=\left[S_{1}, S_{2}, S_{3}\right]$ and characterized by a fixed input SOP interacts nonlinearly with its own counter-propagating replica $\boldsymbol{J}=\left[J_{1}, J_{2}, J_{3}\right]$ through a cross-polarization interaction. The forward signal $\boldsymbol{J}$ is generated at the fiber-end by 
means of an amplified reflective device incorporating a time-delay (in practice, this optical delay is made of a second segment of standard fiber $L d$ for which the propagation regime is supposed to be linear). The dynamics of the system, in particular the chaotic regime, is mainly driven by the amplification factor $g$ of the reflective apparatus defined as the power ratio between the backward and forward signals measured in $z=L: g=|\boldsymbol{J}(L, t)| /|\boldsymbol{S}(L, t)|$. The normalized unitary vectors $\boldsymbol{s}=\boldsymbol{S} /|\boldsymbol{S}|$ and $\boldsymbol{j}=\boldsymbol{J} /|\boldsymbol{J}|$ indicate their corresponding SOPs. For such a system, the spatio-temporal dynamics of $\boldsymbol{S}$ and $\boldsymbol{J}$ are described by the following set of coupled nonlinear propagation equations $[26,30]$ :

$$
\left\{\begin{array}{l}
c^{-1} \partial_{t} \boldsymbol{S}+\partial_{z} \boldsymbol{S}=\gamma S \wedge(D \boldsymbol{J})-\alpha \boldsymbol{S} \\
c^{-1} \partial_{t} \boldsymbol{J}-\partial_{z} \boldsymbol{J}=\gamma \boldsymbol{J} \wedge(D \boldsymbol{S})-\alpha \boldsymbol{J}
\end{array}\right.
$$

where $z$ and $t$ denote the propagation distance and time coordinates, respectively, $c$ is the speed of light, $\gamma$ the nonlinear Kerr coefficient, $D$ the diagonal matrix $\operatorname{diag}(-8 / 9,-8 / 9,8 / 9)$ and $\alpha$ the fiber losses, while the symbol $\wedge$ denotes the vectorial product. Since typical temporal fluctuations come in the microsecond scale, only continuouswaves are considered in simulations, thus the chromatic dispersion has been neglected.

In order to simulate such a system including the time-delayed feedback, we implement the following numerical procedure. Equations (1) are numerically resolved along the whole propagation distance $L+L d$ while the boundary conditions and time-delay are taken into account in such a way that $\boldsymbol{J}(L+L d, t)=g R \boldsymbol{S}(L+L d, t)$, where $R$ denotes the rotation matrix of the reflective device, while the nonlinear Kerr coefficient $\gamma$ as well as fiber losses are taken to zero along the $L d$ segment. In this way, the nonlinear coupling between the counter-propagating waves is only effective for $z=[0, L]$.

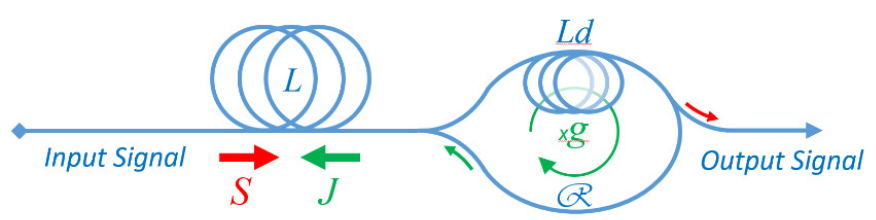

Fig. 1. Principle of operation: The signal wave $S$ is injected and interacts in the fiber of length $L$ with its backward replica $\boldsymbol{J}$ generated by an amplified reflective delayed loop $R$ (with gain $g$ and a delay determined by the fiber length $L d$ ).

Before describing the experimental and numerical results, we would like here to briefly comment by means of simple qualitative arguments the mechanism underlying the fast process of polarization scrambling. As will be discussed below, polarization scrambling is characterized by a fast and disordered motion of the Stokes vectors on the surface of the Poincare sphere. This scrambling process originates from the fact that even weak polarization fluctuations present in the incident waves are magnified through the nonlinear coupling that exists between the wave itself and its counter-propagating amplified replica. In fact, this feedback avalanche process prevents the Omnipolarizer to reach a stationary solution, leading to large polarization temporal fluctuations at its output. Moreover, the insertion of an optical delay within the feedback loop allows to achieve a complete decorrelation between the incident and backward wave fluctuations, which greatly helps the system to enter into its chaotic operation regime. On the other hand, from a fundamental point of view, a key property of Eqs. (1) is that, at variance with usual nonlinear Schrödinger equations governing light propagation in optical fibers, here the dynamics is dominated by the counter-propagating configuration of the interaction, i.e., there are no second-order dispersion effects in Eqs. (1). 
This property introduces an undetermined sign into the expression of the kinetic energy of the waves (setting $\alpha=0$ the Hamiltonian of Eqs. (1) is not bounded from above or below [31]). As a consequence, the system can exhibit a fast decoherence process by creating rapid spatial fluctuations in the motion of $\boldsymbol{S}$ and $\boldsymbol{J}$, because the local increase of kinetic energy due to such rapid fluctuations in the forward $\boldsymbol{S}$ component can be compensated by a corresponding negative reduction in the backward wave component $\boldsymbol{J}$ (the detailed microscopic fluctuations of the backward waves still being decorrelated) [31]. Moreover, in the limit of a conservative interaction of Eqs. (1), such a fast scrambling process was shown to be responsible for an unexpected process of "unconstrained thermalization": At variance with standard thermalization to equilibrium, here the system can freely increase the amount of disorder, because such an increase is no longer constrained by energy conservation, and can thus occur much faster than a slow conventional thermalization process [31].

\section{Experimental setup}

In order to study the chaotic dynamics of that system, we have implemented the experimental setup depicted in Fig. 2. A 1-km length of HNLF is used as nonlinear Kerr medium. The HNLF is characterized by a nonlinear coefficient $\gamma=9 \mathrm{~W}^{-1} \mathrm{~km}^{-1}$ and fiber losses $\alpha$ of 0.7 $\mathrm{dB} / \mathrm{km}$. The HNLF is then encapsulated between two optical circulators. The input signal is amplified using an Erbium doped fiber amplifier (EDFA-1), then the first circulator allows to inject the incident fully-polarized light into the fiber and reject the counter-propagating replica simultaneously. At the opposite end of the HNLF, the time-delayed feedback apparatus consists in a fiber loop made of the second circulator, a 90:10 tap coupler to extract the output signal, a kms long spool of standard single mode fiber (SSMF) as additional delay line and a second amplifier (EDFA-2). The gain of the EFDA-2 is carefully controlled to adjust the amplification factor $g$. A polarization controller is also inserted within the loop in order to control the polarization rotation $\mathrm{R}$ of the reflected beam. For fundamental studies, the input signal consists in a fully-polarized $100-\mathrm{GHz}$ bandwidth incoherent wave centered at $1550 \mathrm{~nm}$. This incident signal is generated from an Erbium-based amplified spontaneous noise source (ASE) sliced into its spectrum domain thanks to an optical filter followed by an inline polarizer. This large bandwidth input signal is used to avoid any impairment due to the stimulated Brillouin backscattering in the fiber under-test but this process is compatible with any type of incident signal. The signal is then amplified up to $14 \mathrm{dBm}$ by means of the EDFA-1 before injection into the fiber. At the output of the system, the SOP of the resulting signal is characterized by means of a standard commercial polarimeter. Furthermore, for random bit generation experiments, the output signal SOP is projected on an inline polarizer in order to transfer the polarization chaos into intensity fluctuations. The resulting random signal is then recorded by means of a $1-\mathrm{GHz}$ photodiode and a fast oscilloscope before digitalization process. 


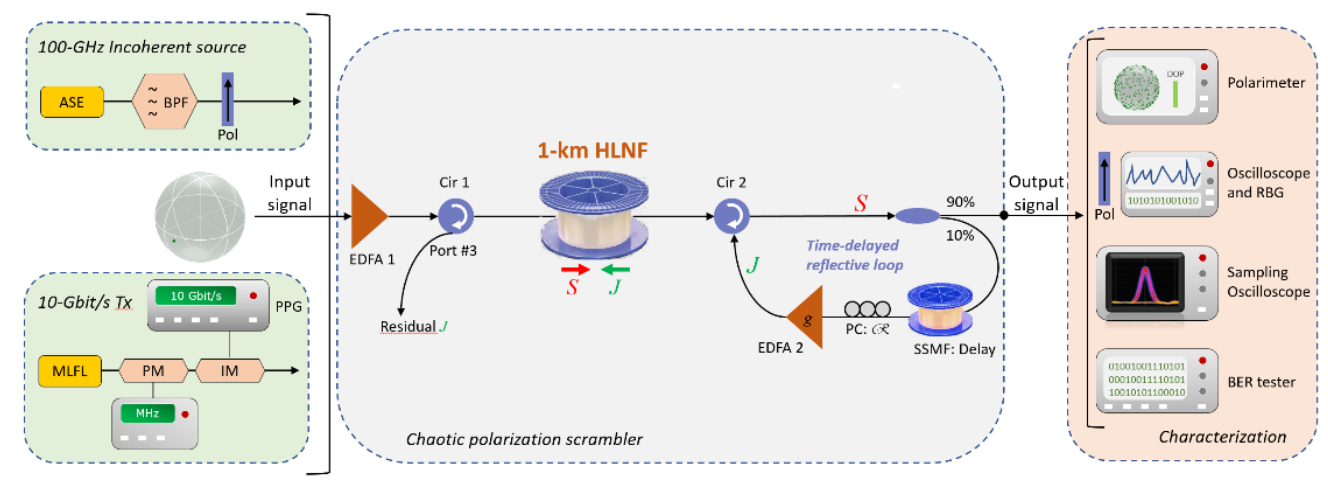

Fig. 2. Experimental setup. ASE: Amplified spontaneous noise emission, BPF: bandpass filter, Pol: Polarizer, PPG: Pulse pattern generator, MLFL: Mode-locked fiber laser, PM: Phase modulator, IM: Intensity modulator, EDFA: Erbium-doped fiber amplifier, CIR: Optical circulator, HNLF: highly nonlinear fiber, PC: polarization controller, SSMF: Standard single mode fiber, RBG: Random bit generation, BER: Bit-error-rate.

In a final step, in order to further assess the performance of this all-optical chaotic scrambler for telecom applications, the incoherent wave has been substituted by a $10-\mathrm{Gbit} / \mathrm{s}$ OOK signal at $1550 \mathrm{~nm}$. This return-to-zero (RZ) optical signal is generated from a $10-\mathrm{GHz}$ mode-locked fiber laser (MLFL) delivering 2.5-ps pulses at $1550 \mathrm{~nm}$. This $10-\mathrm{GHz}$ pulse train is then intensity modulated thanks to a LiNbO3 Mach-Zehnder modulator driven by a $10-\mathrm{Gbit} / \mathrm{s}$ pulse pattern generator (PPG). Note that the initial pulse train is also phase modulated at $100 \mathrm{MHz}$ in order to prevent any deleterious effect from Brillouin backscattering.

\section{Experimental results}

Figure 3(a) displays a 3-dimensionnal operation diagram of our system, which is recorded at the system output as a function of the amplification factor $g$. More precisely, it corresponds to the projection of the output $\mathrm{SOP}$ in the $\mathrm{S}_{2}-\mathrm{S}_{3}$ plane. For that measurements, the input power is fixed to $14 \mathrm{dBm}$ while the optical delay-line for the forward signal consists in a $5-\mathrm{km}$ long spool of SSMF. Three different regions for the $g$ parameter can be observed. First-of-all, for a moderate level of backward power $(g<8, \sim 20 \mathrm{dBm})$, it can be clearly seen that the two waves do not interact, consequently, the output SOP remains almost constant. For higher values of $g$, typically $(8 \leq g<20, \sim 24 \mathrm{dBm})$, the system becomes unstable and starts to oscillate. In this transient regime, more or less complicate close trajectories can be observed whose complexity and frequency of appearance increase with the level of backward power. Unstable fixed points can be also observed. Moreover, in this transient regime, the dynamics of the system was found to be dependent on both the input SOP and the rotation matrix $R$. In contrast, by increasing further the $g$ values beyond 20 allows the system to enter into the chaotic regime. In this case large fluctuations and aperiodic chaotic behaviors are observed independently of the input SOP and the rotation matrix $R$, leading to a full scrambling of the output SOP. In order to illustrate the scrambling and chaotic behavior of the output SOP, we have compared in Figs. 3(b)-3(e) the output Poincare sphere for different values of the amplification factor. While the output signal is characterized by a fixed SOP for a weak value of $g=1$ (a single point on the sphere in Fig. 3(b)), we can clearly note in Fig. 3(c) that the output SOPs describe close trajectories in the transient regime (here $g=10$ ) and then exhibit a more or less complex behavior for larger values of the reflective coefficient $(g=17$ in Fig. 3(d)), before covering almost homogeneously the complete surface of the sphere for high values of $g(g=53$ in Fig. 3(e)). This demonstrates the scrambling potential of the underlying process. To go deeper into the analysis, we have also reported in Figs. 3(b)-3(e) the corresponding RF spectra of the output $S_{1}$ Stokes component. As the reflection coefficient $g$ is 
increased, one can easily notice that the resulting spectrum evolves from a DC component to well-defined set of discrete frequencies in the transient regime, until reaching a broad continuum of frequencies without any discrete lines (Fig. 3(e)), which further evidences the aperiodic and random nature of SOP fluctuations in the chaotic regime. Moreover, to further understand the key role of the time-delay introduced into the reflective loop as well as its amplification factor $g$, we have carried out a series of experiments and numerical simulations in three different configurations for a fixed feedback delay: 0 delay (Omnipolarizer configuration reported in [24]), $1 \mathrm{~km}$ of SSMF included into the reflective loop and then 5 $\mathrm{km}$. Furthermore, we have carefully recorded 100 realizations for each value of the parameter $g$, each of them having a different polarization rotation matrix $R$ to ensure the chaotic regime is reached independently of the SOP of the backward replica. The input power is still kept constant to $14 \mathrm{dBm}$. The scrambling performances have been evaluated by means of the degree-of-polarization (DOP) and scrambling speed $\left(V_{s c r}\right)$ defined as:

$$
\begin{gathered}
\mathrm{DOP}=\frac{\sqrt{\left\langle S_{1}(t)\right\rangle^{2}+\left\langle S_{2}(t)\right\rangle^{2}+\left\langle S_{3}(t)\right\rangle^{2}}}{\left\langle S_{0}(t)\right\rangle} \\
V_{s c r}=\left\langle 2 \arcsin \left(\frac{1}{2} \frac{\partial \vec{s}(L, t)}{\partial t}\right)\right\rangle
\end{gathered}
$$

Figures 4(a)-4(c) summarize the experimental results for the output DOP as a function of $g$, while Figs. 4(d)-4(f) report the corresponding scrambling speed $V_{s c r}$. Red solid lines represent average values of DOP and speed, while the shaded areas display respective fluctuations (standard deviation in grey and maximum excursion in ochre). 

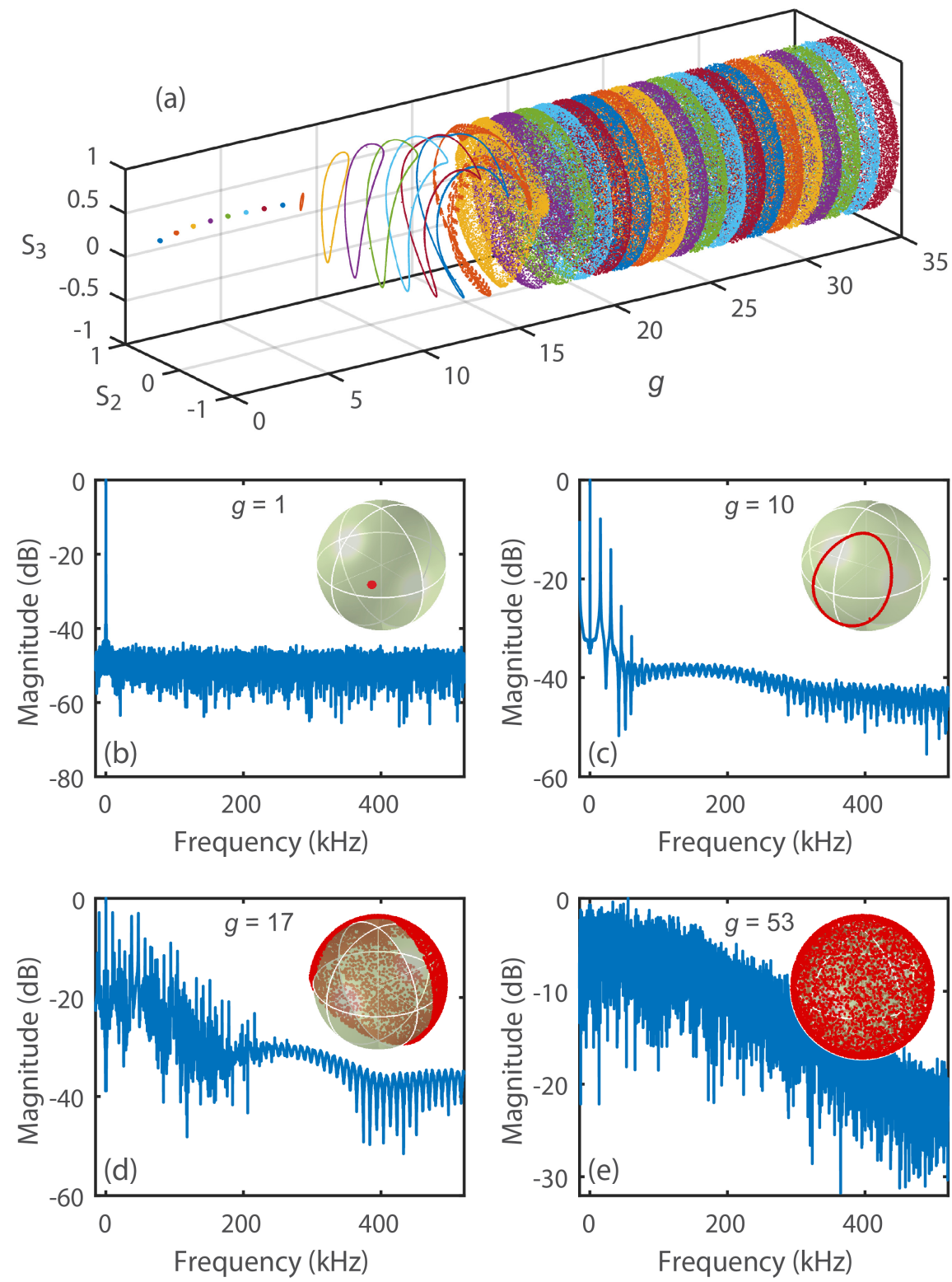

Fig. 3. (a) Bifurcation diagram of the system: Projection of the experimentally measured output SOP in the S2-S3 plane (b-e) Poincare sphere for different values of the reflective amplification factor $g$ as well as corresponding RF spectrum of the $S_{l}$ component: (b) $g=1$, (c) $g=10,(\mathrm{~d}) g=17$ and (e) $g=53$.

From Figs. 4, the influence of the delay-line becomes evident. Indeed, for an increasing delay starting from 0 to $5 \mathrm{~km}$, the threshold value of $g$ required to enter into a genuine chaotic scrambling regime and thus reach a DOP close to zero (at least $<5 \%$ ) is found to be significantly reduced. More precisely, compared to our previous works (here zero delay case in Fig. 4(a)), the insertion of a delay-line within the reflective loop allows to achieve at least a 2.5 -fold reduction of the chaotic amplification factor threshold. Moreover, it is important to stress that the strong system fluctuations, typically generated in the transient regime, progressively vanish for $g \geq 40$ when a $1-\mathrm{km}$ long delay-line is inserted in the system and just above 30 (backward power of $26 \mathrm{dBm}$ ) with a $5-\mathrm{km}$ spool of delay, thus making the 
scrambling performances more predictable and reliable. The same behavior can be observed for the scrambling speed in Figs. 4(d)-4(f) for which at least a 2-fold increase of $V_{s c r}$ is achieved for the same amplification factor value when a 5-km long delay-line is inserted in the system. The performances and repeatability of the device are also greatly improved with a large reduction of fluctuations in the output scrambling speed. These results underline the fact that the polarization instability and its route to chaos can be significantly improved by feeding this self-organized system with an optical time-delay. We attribute this behavior to the fact that the polarization fluctuations of the backward waves are mutually decorrelated from each other, which greatly helps the system to enter into the chaotic regime. Finally, we have also reported in Figs. 4 by means of black circles the results obtained from numerical resolution of Eqs. (1) averaged over 24 realizations, each involving a different rotation matrix $R$ and including the exact experimental parameters, in particular taking into account for the limit response of our polarimeter $(1 \mathrm{Msa} / \mathrm{s})$. We can observe an excellent agreement between our numerical predictions and the experimental data, thus validating our theoretical model and providing a reliable tool for designing this home-made chaotic polarization scrambler.
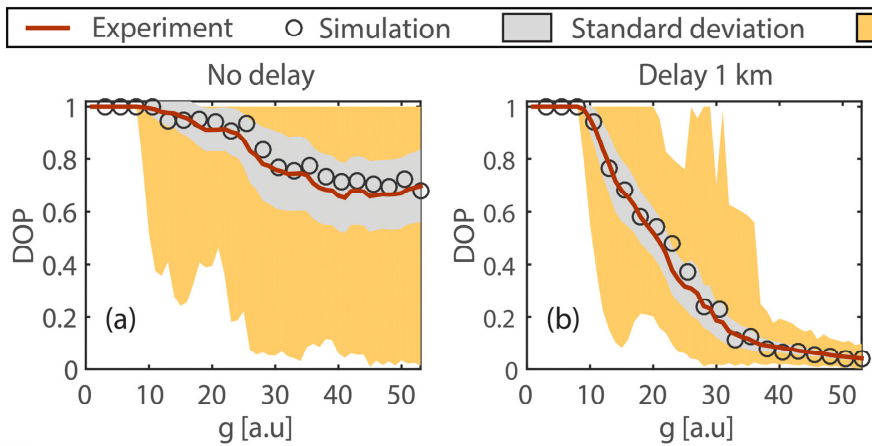

Maximum excursion
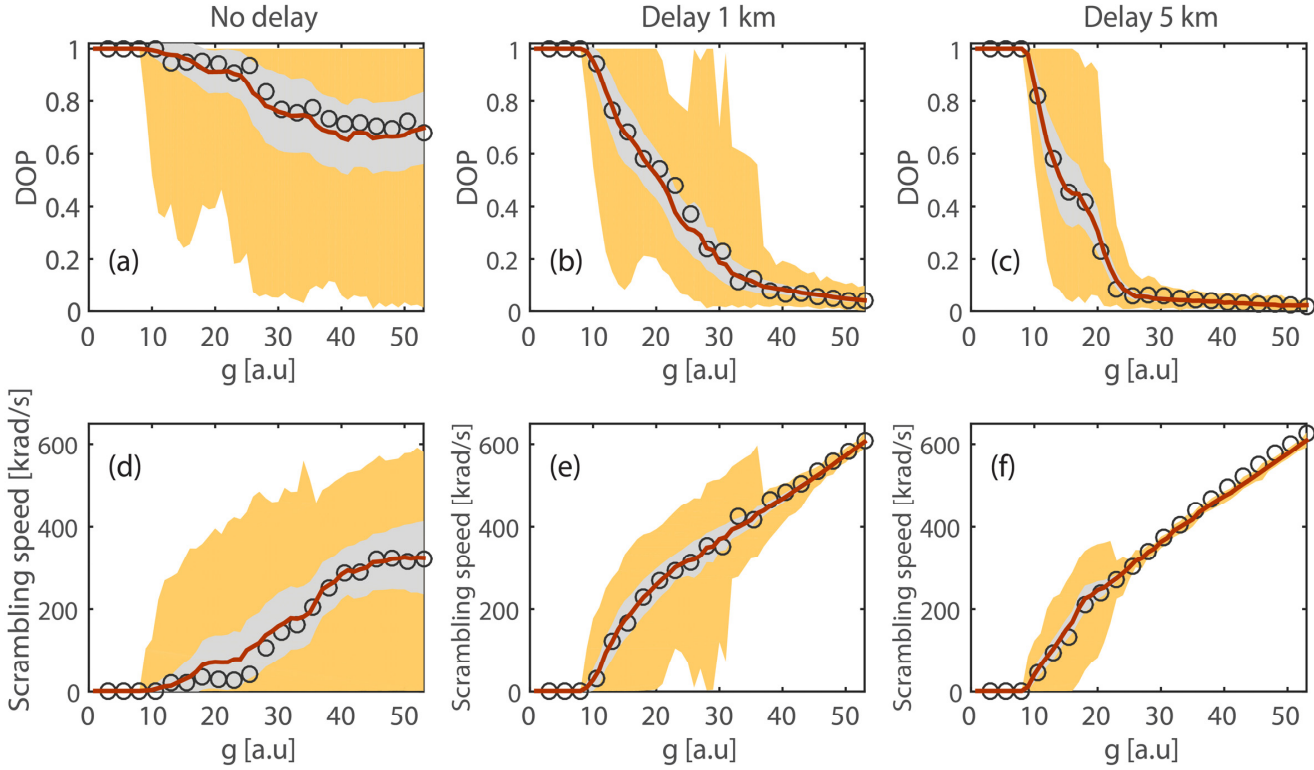

Fig. 4. (Top line) DOP measured at the output of the system as a function of the amplification factor $g$ for the 0-delay configuration (a) a 1-km long delay-line (b) and 5-km long delay-line (c), respectively. (Bottom line) Scrambling speed as a function of amplification factor $g$ (same convention as for DOP measurements). The red solid-lines correspond to the experimental data averaged over 100 realizations, while the shaded areas represent respective fluctuations (standard deviation in grey and maximal excursion in ochre). The black circles correspond to the numerical simulation results averaged over 24 realizations.

In order to highlight in more details the key role played by the delay-line, we have carried out the following additional measurements. For a fixed maximal amplification factor of $g=$ 53, we have measured the resulting scrambling speed and DOP at the output of the Omnipolarizer as a function of the fiber length inserted into the reflective loop. Moreover, to further characterize the chaotic nature of our system, we have also calculated the corresponding Lyapunov coefficient $L$ following the procedure described in [32]. These measurements are summarized in Figs. 5, and have been averaged over 100 realizations involving different rotation matrix $R$. 
We can fairly observe the strong impact of the feedback delay-line with a clear threshold around $200 \mathrm{~m}$ which enables to reach the maximum scrambling speed near $610 \mathrm{krad} / \mathrm{s}$ (blue circles) and very low values of DOP (red triangles). This threshold behavior is consistent with the nonlinear response of the system and can be explained by the fact that, to enter into a fully chaotic regime, the decorrelation time-scale between both counter-propagating waves is governed by the nonlinear length of the system defined by $1 / \gamma P$, leading to $180 \mathrm{~m}$ for $g=$ 53. Note also the excellent agreement between our measurements and numerical predictions (averaged over 24 runs), depicted in Fig. 5(a) by means of blue stars for the scrambling speed and red crosses for DOP. Finally, the calculation of the experimental Lyapunov coefficient $L$, depicted here in Fig. 5(b), shows that thanks to the inclusion of a delay-line in the system, $L$ becomes largely positive for a fiber length beyond $200 \mathrm{~m}$ when $g=53$, confirming aforementioned conclusions and providing a clear signature of the chaotic nature of the SOP at the output of the system. As already highlighted in Fig. 4, we can also notice the strong reduction of fluctuations in the system performance owing to the feedback delay, thus making our device more reliable. Finally, Fig. 5(c) displays the typical temporal evolution of the $S_{I}$ Stokes parameter at maximum scrambling speed and for $5 \mathrm{~km}$ of delay, directly recorded at the output of the fiber beyond a polarizer. We can clearly see that typical temporal fluctuations come in the microsecond scale.
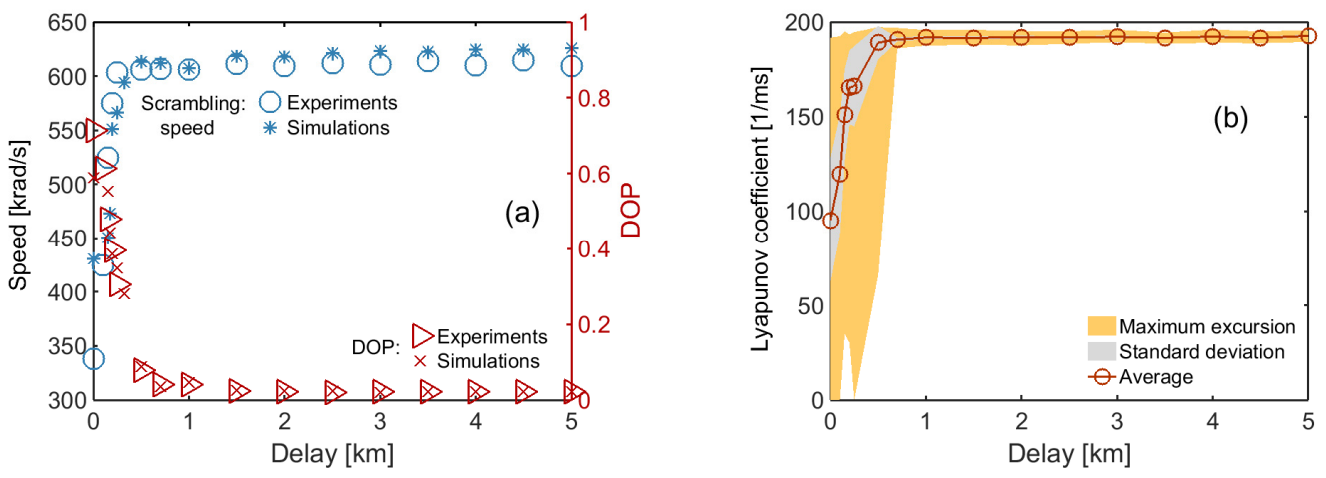

Fig. 5. (a) Scrambling speed (blue circles) and corresponding DOP (red triangles) as a function of fiber length used as delay-line within the reflective loop of the Omnipolarizer. The amplification factor $g$ is kept constant to $g=53$. Numerical simulations are displayed with blue stars and red crosses, respectively (b) Corresponding experimental Lyapunov coefficient L as a function of the fiber length inserted into the reflective loop. All measurements have been averaged over 100 realizations (c) Typical temporal evolution of the $S_{I}$ parameter at maximum scrambling speed and $5 \mathrm{~km}$ of delay.

\section{Polarization scrambling of a $\mathbf{1 0}-\mathrm{Gbit} / \mathrm{s}$ optical signal}

The chaotic all-optical scrambler under-study has been tested in a telecommunication configuration. For this proof-of-principle, the partially coherent wave $(100-\mathrm{GHz})$ described in the first section has been substituted with a 10-Gbit/s RZ signal centered at $1550 \mathrm{~nm}$. The pulse width has been chosen as short as $2.5 \mathrm{ps}$ in order to evaluate the impact of the scrambler for higher repetition rates of data or high-frequencies analogic signals. The delay-line inserted into the reflective loop is fixed to $5 \mathrm{~km}$ while the input power is kept constant to $14 \mathrm{dBm}$. To ensure that our device operates in a genuine chaotic regime, the amplification factor has been chosen to $g=53$, corresponding to a backward power of $28 \mathrm{dBm}$. Figure 5 summarizes our results. Firstly, Figs. 6(a) and 6(b) display respectively the input and output Poincaré sphere of the 10-Gbit/s signal. While the input SOP is totally fixed at the input of the system, the output Poincaré sphere appears entirely covered, thus confirming that an efficient scrambling process can be achieved, even with high-repetition rate pulsed signals. Furthermore, Figs. 6(c) and 6(d) depict the output eye-diagrams when the backward signal is OFF (Fig. 6(c)) and in 
scrambling configuration (Fig. 6(d), pump ON). We can observe that the shape of the pulses is ideally preserved with a clearly opened output eye-diagram, which validates the applicability of our polarization scrambler to RZ telecom signals. Note however the presence of an additional amplitude jitter into the scrambled eye-diagram in Fig. 6(d). This source of noise was attributed to the Rayleigh backscattering imposed by the backward signal on the output beam. Nevertheless, in a wavelength division multiplexing configuration, one could exploit one isolated pump channel in order to filter out this deleterious noise source, as already proposed in [24]. To further assess the quality of the transmitted signal, we have performed bit-error-rate (BER) measurements as a function of the average power incoming on the receiver. Figure 6(e) compares the back-to-back configuration (blue circles) with the pump OFF (red dots) and ON (purple diamonds) cases for two values of the backward power ( $29 \mathrm{dBm}$ and $30 \mathrm{dBm})$. We can first stress that an error-free transmission is achieved for the scrambled signal, confirming that our scrambler is fully compatible with such single-channel RZ telecom configuration. However, a slight power penalty $(0.5 \mathrm{~dB})$ has been measured between the pump ON/OFF curves, which is attributed to the Rayleigh backscattering induced by the intense backward signal. A power penalty of $1.5 \mathrm{~dB}$ has been detected compared to the input configuration, which is mainly attributed to the deleterious effects of chromatic dispersion and Kerr effect on the ultra-short pulses used in our experiments.
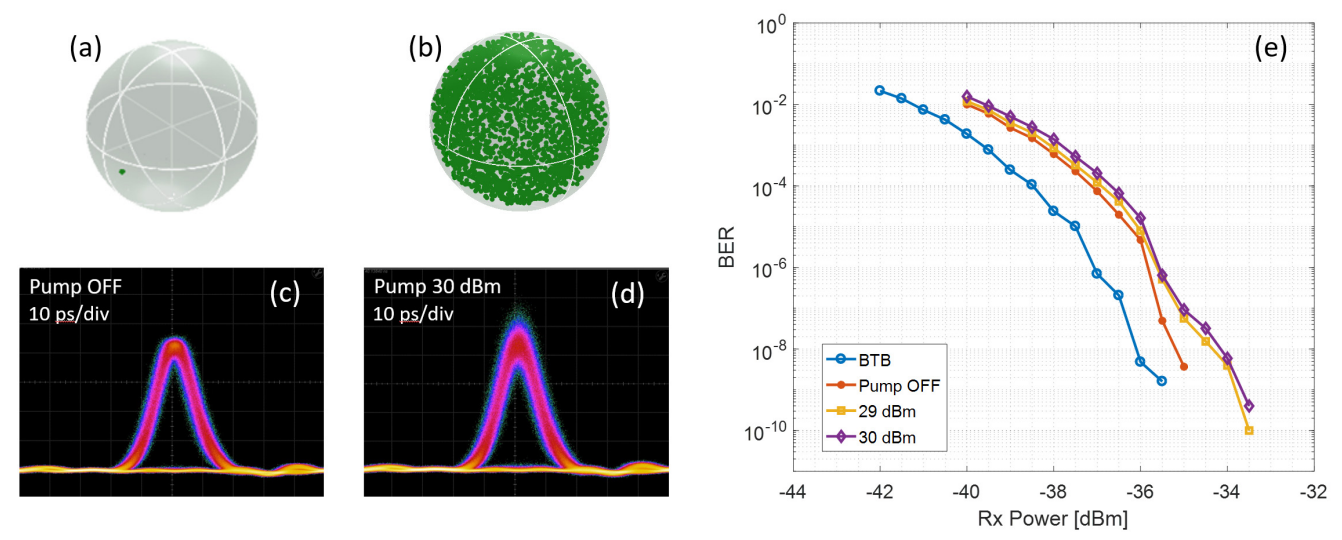

Fig. 6. (a) Input Poincaré sphere of the 10-Gbit/s RZ signal (b) Output SOP in scrambling regime for $g=50(\mathrm{c} \& \mathrm{~d})$ Output eye-diagrams of the 10-Gbit/s signal recorded in pump OFF (c) and pump ON configuration (d) (e) BER measurements as a function of the average power incoming on the receiver, back-to-back configuration (blue circles), pump OFF (red dots) and pump ON: $29 \mathrm{dBm}$ (yellow squares) and $30 \mathrm{dBm}$ (purple diamonds).

\section{Random bit generation}

The genuine chaotic nature of our all-optical scrambler provides a good opportunity to exploit this optical system as RNG, a field that has recently received much attention in photonics [613]. To this aim, in the last section of this manuscript, we take advantage of the chaotic evolution of the output Stokes parameters to experimentally generate random bit sequences. For this proof-of-concept experiment, the evolution over time of a Stokes parameter has been recorded and concatenated from a large number of different realizations in order to construct a $10^{9}$ bit sequence. Such long sequences are mandatory in order to perform standard statistical Dieharder tests as described below [29]. The principle of operation of random bit generation from polarization chaos is to convert a Stokes component of the output field of the scrambler into either a 0 or 1 depending on its relative value according to some specific threshold, here calculated from the median value of the waveform. In this series of experiments, the $S_{I}$ component is recorded beyond an inline polarizer by means of a photo-receiver connected to a $1-\mathrm{Gsa} / \mathrm{s}$ oscilloscope, $10^{6}$ slots of $100-\mathrm{ms}$ each were then acquired, concatenated and sampled. For that purpose, the chaotic polarization scrambler uses a $5-\mathrm{km}$ delay-line as well 
as an amplification factor $g=53$. The input signal corresponds to the $100-\mathrm{GHz}$ partially coherent wave described in the first section of the paper. Figure 7(a) displays a part of a typical experimental realization of the raw data (blue solid-line) as well as the post-processing involved in the random numbers extraction. Basically, the waveform is first under-sampled at a clock rate whose frequency is chosen well below the typical correlation length of the signal under test so as to ensure a reliable randomization. In our practical case, the clock (in black) has been chosen to $10 \mathrm{kHz}$ for a typical scrambling speed of $610 \mathrm{krad} / \mathrm{s}$. The $S_{I}$ signal is then sampled (red points) at each rising edge of the clock. After suitable thresholding by calculating the median value of the sampled signal, the binary random sequence represented in Fig. 7(b) has been obtained. Note that 5 days of continuous recording involving more than 1.2 To of raw data have been necessary to construct the billion bit sequence required for the dieharder test. Finally, in order to improve the randomness of the sequence and successfully pass the benchmark tests, we also remove any residual correlation and bias associated with binary conversion using an exclusive-or (XOR) gate between the initial sequence and its time-delayed replica $[6,9,12,13]$. A delay of 100 bits was here applied. The degree of randomness of the computed binary sequences are first tested through calculation of the autocorrelation trace (Fig. 7(c)), and the cross-correlation function between two different sequences (Fig. 7(d)). These first results reveal a vanishing cross-correlation for all of the generated random bit streams, indicating that all the sequences are different and that the proposed technique is a good candidate for RNG.

(a)

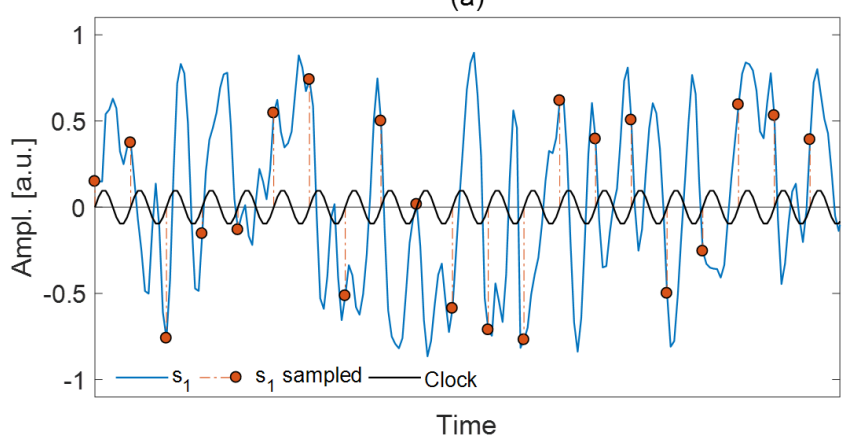

(b)

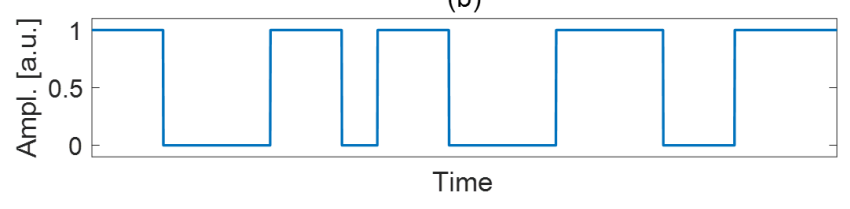

(c)

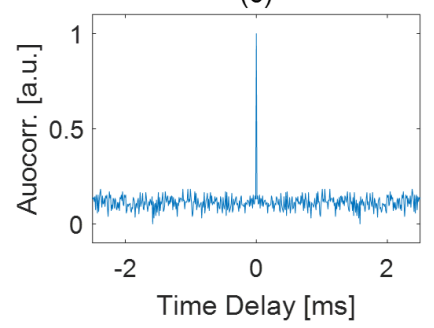

(d)

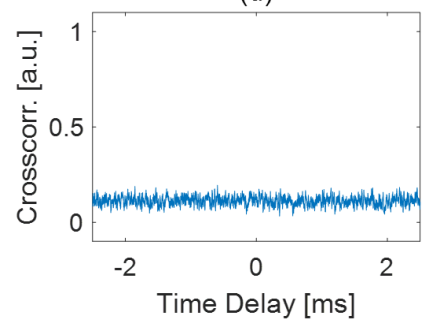

Fig. 7. (a) In blue, evolution of the $S_{I}$ parameter over time. In red sampled values taken in correspondence of rising edges of an ad hoc defined clock (in black). (b) Random sequence generated after thresholding samples chosen in (a). In (c) and (d) autocorrelation and crosscorrelation functions of different random sequences generated in experiments.

To further assess the degree of randomness of the generated billion bit sequence, we have implemented the standard statistical benchmark dieharder tests. Results are summarized in Fig. 8 [see also Dataset 1 (33) for complete results] and show that the generated sequences pass all the most commonly used statistical tests (p-value $>0.01$ ), thus demonstrating that the polarization chaos induced by a counter-propagating time-delayed feedback is a suitable source of randomness to generate random binary sequences. It is important to note that the Dieharder suite also includes all the statistical tests developed by the National Institute for Standards and Technology (NIST), as well as some extra tests [29], which all successfully 
passed with resulting values between 0.04 and 1 , thus demonstrating the genuine randomness of the generated sequence.

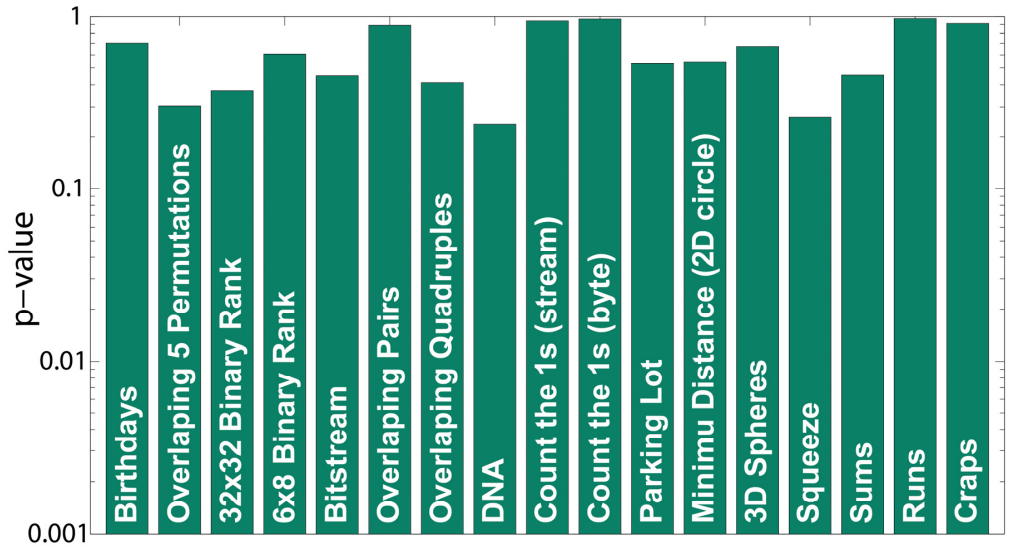

Fig. 8. Dieharder benchmark tests results for the $10^{9}$ experimental binary sequence generated from polarization chaos.

\section{Conclusions}

In summary, we have experimentally investigated the chaotic dynamics of the state-ofpolarization in a nonlinear optical fiber fed by an intense time-delayed backward replica. The fundamental basis of this work was initially proposed in [24] as an additional working regime of the device called Omnipolarizer [26] and dedicated to polarization scrambling. This system relies on a nonlinear cross-polarization interaction occurring in an optical fiber between an incident signal and its own high-power counter-propagating replica, generated at the fiber-end through an amplified reflective loop apparatus. In this new contribution, we go significantly beyond these previous results and show that the polarization instability and its route to chaos can be significantly improved by feeding this self-organized system thanks to a time-delayed feedback. Indeed, the polarization fluctuations of the incident and reflected waves are completely decorrelated from each other, which significantly helps the system to enter into a chaotic regime. For that particular configuration, we have shown that the instability threshold and transient regimes can be substantially reduced when a delay-line is incorporated in the reflective loop, with a time scale longer than the nonlinear characteristic time of the system. We have then reported on a series of experiments involving a 1-km long HNLF-based polarization scrambler including different lengths of delay-line within the reflective loop, which revealed that scrambling speeds up to $600-\mathrm{krad} / \mathrm{s}$ can be achieved. A 2.5 -fold reduction in terms of power threshold compared to our previous observations as well as a significant reduction of the system performance fluctuations in the scrambling speed and output DOP have been observed. The performance of this all-optical scrambler for telecom applications has been evaluated on a 10-Gbit/s OOK RZ signal with error-free transmission. Finally, we also described how the chaotic nature of polarization fluctuations can be exploited to generate ensembles of random bit sequences. More precisely, through a digitalization process of the Stokes parameters at the output of the system, we have experimentally generated a billion bit sequence at a repetition rate of $10 \mathrm{kHz}$ whose randomness has successfully passed the benchmark dieharder test. The speed of our random bit generator is quite low compared to recent publications, which can achieved several of Gbit/s in $[6,7,12,13]$. However, these results represent the first proof-of-principle demonstration of random bit generation through a counter-propagating cross-polarization interaction. Moreover, the speed of our system as well as its compactness could be improved by implementing ultra-high nonlinear materials or high-confinement waveguides such as soft-glass optical fibers or silicon waveguides 


\section{Optics EXPRESS}

integrated on a CMOS compatible chip in order to achieve the Gbit/s repetition rate. Finally, our experimental observations have been well confirmed by numerical simulations based on the resolution of counter-propagating coupled nonlinear propagation equations.

\section{Funding}

European Research Council (ERC Starting Grant PETAL) (306633); Agence Nationale de la Recherche (ANR) (APOFIS); Labex Action (ANR-11-LABX-0001-01); FEDER; Conseil Régional de Bourgogne Franche Comté.

\section{Acknowledgments}

We thank Dr. Daniel Brunner from Femto-st institute in Besancon, as well as Dr. Anthony Martin from the University of Geneva for fruitful discussions. 